Résumés des conférences et travaux

\title{
Histoire des sciences au Moyen Âge
}

Danielle Jacquart

\section{OpenEdition \\ Journals}

Édition électronique

URL : https://journals.openedition.org/ashp/1001

DOI : 10.4000/ashp.1001

ISSN : 1969-6310

Éditeur

Publications de l'École Pratique des Hautes Études

\section{Édition imprimée}

Date de publication : 2 février 2011

Pagination : 165-167

ISSN : 0766-0677

\section{Référence électronique}

Danielle Jacquart, " Histoire des sciences au Moyen Âge », Annuaire de l'École pratique des hautes études (EPHE), Section des sciences historiques et philologiques [En ligne], 141 | 2011, mis en ligne le 24 février 2011, consulté le 06 juillet 2021. URL : http://journals.openedition.org/ashp/1001 ; DOI : https://doi.org/10.4000/ashp.1001 


\title{
HISTOIRE DES SCIENCES AU MOYEN ÂGE
}

\author{
Directeur d'études : $\mathrm{M}^{\mathrm{me}}$ Danielle JACQUART
}

Programme de l'année 2008-2009 : I. L'intérêt pour les sciences durant le haut Moyen Âge. II. Les transformations de la matière et leurs théories médiévales.

\section{L'intérêt pour les sciences durant le haut Moyen Âge}

En une première séance d'introduction générale, le directeur d'études a pris pour fil conducteur un article de Jean Dhombres récemment paru («René Taton et les questions posées par l'histoire professionnelle des sciences », Archives internationales d'histoire des sciences, 57, 2007, p. 363-382). Dans cet article à la provocation stimulante, l'auteur incite l'historien des sciences à s'intéresser au « mobile »: « Reste encore la vulgaire question du mobile, la seule qui compte, et pas seulement dans un roman policier : qu'est-ce qui fait courir le savant? [...] L'histoire des sciences post-moderne élude la question de la libido sciendi» (p. 379). Pour les siècles si peu « scientifiques » que couvre le haut Moyen Âge, la recherche des motivations des lettrés qui se sont intéressés aux mathématiques, à l'astronomie ou à l'étude de quelque phénomène naturel est en effet centrale, même si elle ne se pose pas dans les mêmes termes que pour la période moderne à laquelle se réfère Jean Dhombres. S'il y eut peu d'œuvres de « science » stricto sensu durant le haut Moyen Âge, des curiosités multiples sont disséminées, avec plus ou moins d'ampleur, dans des ouvrages aux propos les plus variés.

Pour respecter une continuité avec les conférences de l'année précédente (Annuaire 2007-2008, p. 176-177), dont une grande partie avait porté sur Jean Scot Érigène, nous avons commencé par rechercher et évaluer les intérêts scientifiques manifestés par Rémi d'Auxerre. À bien des égards, cet auteur peut être considéré comme un passeur entre l'univers des lettrés carolingiens et celui des écoles cathédrales tel qu'il préfigure les habitudes d'enseignement qui allaient se cristalliser dans les futures universités. Dans son commentaire au De nuptiis Philologiae et Mercurii de Martianus Capella, Rémi d'Auxerre reprend, certes, des passages des gloses de Jean Scot Érigène, mais son œuvre est plus ample, moins technique et plus pédagogique. La lecture de son commentaire nous a menés à la conclusion qu'il ne fallait pas se contenter d'analyser les parties explicitement centrées sur le quadrivium, mais que même dans les premiers livres, qui exposent l'intrigue, n'importe quel mot pouvait introduire un développement sur tel ou tel phénomène naturel. Les intérêts scientifiques sont ainsi disséminés tout au long du commentaire. Nous nous sommes particulièrement attachés aux deux couples astrologia-geometria et astronomia-arithmetica et avons remarqué dans le premier une référence plus nette à la géométrie pratique que chez Jean Scot Érigène. En outre, une lecture conjointe du commentaire de Rémi d'Auxerre aux premiers versets de la Genèse a mis en évidence un réel intérêt pour l'astrologie des mathematici.

Le démarcage que fait Rémi d'Auxerre, dans son interprétation de ces versets de la Genèse, du commentaire de Bède le Vénérable, à travers les emprunts effectués par 
Raban Maur, nous a incités à revenir sur ce dernier auteur. Auparavant un retour en arrière sur la tradition isidorienne a été jugé nécessaire. Une séance a donc été consacrée au De natura rerum, dont la version longue, composée dans les écoles northumbriennes, est parvenue au monastère de Fulda, où Raban Maur a reçu sa formation initiale et où il occupa la charge d'abbé entre 822 et 844 . Du De natura rerum d'Isidore de Séville, c'est surtout le chapitre sur les éléments qui nous a retenus, du fait qu'il permettait d'établir un lien avec le second thème mis au programme de l'année. De la tradition isidorienne, la lecture du De universo ou De rerum natura de Raban Maur fait surtout apparaître des reprises littérales des Étymologies. Après avoir comparé la structure d'ensemble des Étymologies et du De universo, nous avons analysé comment dans cette dernière œuvre, dont le propos ne s'écarte guère d'une vocation apologétique et d'édification chrétienne, les interprétations des phénomènes naturels secundum historiam et secundum allegoriam étaient, contrairement à la manière dont avait procédé Isidore de Séville dans son De natura rerum, étroitement entremêlées.

Pour terminer ce parcours à travers les textes du haut Moyen Âge, une séance a été consacrée, en une brève évocation, à Abbon de Fleury. Le point de départ était fourni par deux publications : les Actes d'un colloque édités par Barbara Obrist (Abbon de Fleury, Philosophie, sciences et comput autour de l'An Mil, Paris - Villejuif, 2004, [Oriens-Occidens, 6]) et le livre de Nadja Germann (De temporum ratione, Quadrivium und Gotteserkenntnis am Beispiel Abbos von Fleury und Hermannus von Reichenau, Leyde - Boston, 2006). Outre le commentaire au Calculus de Victorinus d'Aquitaine, qui révèle surtout un intérêt pour le symbolisme des nombres, c'est le recueil de comput qui a retenu notre attention, avec sa particularité d'être centré sur la visualisation et d'être composé essentiellement de tables et de figures. Afin d'établir un lien avec l'étude du De temporum ratione de Bède le Vénérable effectuée deux ans auparavant (Annuaire 2006-2007, p. 155), nous avons particulièrement analysé le diagramme en forme de roue sur les marées. Sur ce sujet Abbon de Fleury se révèle moins précis que Bède auquel il se réfère explicitement. De manière générale, comme tente de le montrer Nadja Germann dans son livre, pour Abbon le quadrivium et l'étude des phénomènes naturels ont pour seul objet de contribuer à la connaissance de Dieu.

Le cycle de trois ans, au cours duquel ont été analysées des œuvres composées entre le $\mathrm{VII}^{\mathrm{e}}$ et le début du $\mathrm{XI}^{\mathrm{e}}$ siècle, a mis en évidence une diversité d'approches des arts du quadrivium et des sciences de la nature. Si toutes ces œuvres s'insèrent peu ou prou dans le cadre d'une explication de la création divine, telle que relatée par le texte biblique, de grandes différences existent d'un auteur à l'autre sur la manière de traiter de ces sujets, certains se dégageant plus que d'autres de la finalité apologétique ou exégétique pour exprimer un véritable intérêt « scientifique » ou naturaliste. Toute généralisation sur la période du haut Moyen Âge doit donc prendre en compte ces différences avec une acuité renouvelée.

\section{Les transformations de la matière et leurs théories médiévales}

Sans perdre de vue le sujet des rapports entre médecine et alchimie, qui avait été traité pendant trois années consécutives (Annuaire 2005-2006, p. 212-215 ; 2006-2007, p. 154-156; 2007-2008, p. 176-179), un nouveau thème a été inauguré. Son propos 
est de suivre dans la continuité chronologique les conceptions relatives aux transformations de la matière, que celles-ci se produisent dans des êtres inanimés ou vivants. Il a paru nécessaire d'inaugurer ce cycle par l'analyse des textes fondateurs, en tenant compte des ponctuations que marquent dans la diachronie les différentes vagues de traductions. Une première ponctuation est formée par les traductions médicales de Constantin l'Africain réalisées à la fin $\mathrm{du} \mathrm{XI}^{\mathrm{e}}$ siècle et par les traductions, à partir du grec, du corpus aristotélicien antérieures à la diffusion de celles effectuées, à partir de l'arabe, par Gérard de Crémone. Au XII ${ }^{\mathrm{e}}$ siècle, ces nouveaux textes furent lus en étroite symbiose avec la tradition du Timée de Calcidius. Plusieurs séances ont donc été consacrées à la notion de matière première (silva) et à la théorie élémentaire dans la traduction et le commentaire de Calcidius, en les mettant en relation avec les conceptions platoniciennes originelles. Nous sommes passés ensuite à la tradition aristotélicienne, avec l'analyse de plusieurs passages du De generatione et corruptione dans la version de Burgundio de Pise et de la Physique dans la version de Jacques de Venise. Bien qu'en cette première étape les traductions de Gérard de Crémone aient été laissées volontairement de côté, une exception a été faite pour le livre I des Météorologiques qui, du moins dans sa première rédaction, semble avoir été assez tôt diffusé. Après ces analyses préalables de la tradition calcidienne et du premier Aristote latin, les séances suivantes ont porté sur les œuvres d'Urso de Salerne (deuxième moitié du XII ${ }^{\mathrm{e}}$ siècle), plus spécialement sur le De commixtionibus elementorum, d'une profonde originalité et à l'influence durable, et plus ponctuellement sur les Glosule à ses propres aphorismes. L'étude de ces œuvres a été centrée sur les notions de matière première, d'élémenté et de mélange, cette dernière notion donnant lieu chez Urso à une théorie des transformations de la matière apte à rendre compte de la diversité de tous les processus naturels, qu'ils appartiennent au monde du vivant ou à l'inanimé, au monde céleste ou terrestre. Il est peu d'auteurs médiévaux qui aient poussé aussi loin leur inventivité pour bâtir un système. Au cours du traitement de ce thème, le directeur d'études a fait un compte rendu détaillé du volume 7 de la revue Quaestio, paru en 2007, dédié à « La matière ». 